\title{
Teste oral com glicose de milho com e sem sódio produzem diferentes curvas de insulina e de glicose em equinos sadios
}

\author{
Oral test with corn glucose with or without sodium produce \\ different insulin and glucose results in healthy horses
}

Keity Laiane Gomes Trindade ${ }^{[a]}$,Virgínia Theodora Brito Marques de Oliveira ${ }^{[a]}$, Maria Eduarda de Oliveira Cavalcanti[a], Micheline Ozana da Silva ${ }^{[b]}$, Helena Emilia Cavalcanti da Costa Cordeiro Manso ${ }^{[a]}$, José Dantas Ribeiro Filho ${ }^{[b]}$, José Mário Girão Abreu[c], Helio Cordeiro Manso Filho ${ }^{[]^{*}}$

\footnotetext{
[a] Núcleo de Pesquisa Equina, Departamento de Zootecnia, Universidade Federal Rural de Pernambuco (UFRPE), Recife, PE, Brasil

[b] Departamento de Medicina Veterinária, Universidade Federal de Viçosa (UFV), Viçosa, MG, Brasil

[c] Faculdade de Medicina Veterinária, Universidade Estadual do Ceará, Fortaleza, CE, Brasil
}

*Autor correspondente

E-mail: helio.mansofo@ufrpe.br

\section{Resumo}

O uso em larga escala dos grãosde cereais processados para equinos tem sido associado à obesidade, síndrome metabólica e às disfunções na microbiota intestinal nessa espécie. Esses trabalhos objetivaram avaliar o teste oral com glicose de milho, com e sem sódio, sobre as concentrações plasmáticas de glicose, insulina e proteínas plasmáticas em cavalos sadios. A hipótese a ser testada é de que a adição de sódio à glicose de milho irá produzir diferentes curvas de glicose e de insulina quando os equinos em jejum, quando comparado com similar produto sem sódio. Foram utilizados seis equinos, distribuídos em um fatorial com três tratamentos (6x3). Os tratamentos foram: 400cal de glicose de milho com sódio (T-GNa400), 200cal de glicose de milho com sódio (T-GNa200), e 400cal de glicose de milho sem sódio (T-G400). Amostras de sangue foram colhidas com os animais nas seguintes fases: pré-teste (jejum) e 0,5, 1,0, 1,5, 2,0, 3,0 e 4,0 horas após a ingestão do tratamento. 0 plasma obtido foi utilizado para determinação da glicose ([GLIC]), insulina ([INSU]) e proteínas plasmáticas totais ([PPT]). Os resultados foram submetidos ao ANOVA com dois fatores (três tratamentos e sete fases) e ao teste deTukey, pelo programa SigmaPlot 13.0, com P estabelecido em $5 \%$. Os resultados indicaram que [GLIC] difere tanto entre os tratamentos como entre as fases $(\mathrm{P}<0,001)$, e que há interação significativa entre os tratamentos e as fases $(P=0,044)$. A [GLIC] foi mais elevada no T-GNa400 ( 113,8 mg/dL), seguido pelo T-G400 ( 106,4 mg/dL) e mais baixa no T-GNa200 (98,5 mg/ $\mathrm{dL})(\mathrm{P}<0,001)$. Também a [INSU]apresentou diferenças entre os tratamentos e entre as fases $(\mathrm{P}<0,001)$, e com interação significativa entre os tratamentos e as fases (P = 0.037). A maior [INSU] foi observada no T-GNa400 ( 18,6 $\mu \mathrm{IU} / \mathrm{mL})$, seguido pelo T-G400 ( 13,1 $\mu \mathrm{IU} / \mathrm{mL})$ e pelo T-GNa200 $(\sim 9,3 \mu \mathrm{IU} / \mathrm{mL})(\mathrm{P}<$ 0,001). Não ocorreram diferenças entre os tratamentos e entre as fases na [PPT]. Conclui-se que a glicose de 
milho, com sódio, durante o teste oral com ingestão produz curvas sanguíneas de glicose e insulina quando comparado com produto sem sódio, quando os equinos estão em jejum, sendo mais elevadas no T-GNa400.

Palavras-chave: Equino. Glicemia. Hormônio. Nutrição.

\section{Abstract}

The use of processed cereals grain in large scale for horses feed had been associated with obesity, metabolic syndrome and intestinal microbiome dysfunction in this specie. This research aim was to evaluate the oral test with corn glucose, with or without sodium over the glucose, insulin and total plasma protein in healthy horses, which was used to test the hypothesis that sodium addition into corn glucose will produce difference in glucose and insulin when compared with similar product without sodium. It was used a latin square design with 6 horses in three treatments (6x3). The treatments were: 400cal of corn glucose with sodium (T-GNa400), 200cal of corn glucose with sodium (T-GNa200) and 400cal of corn glucose without sodium (T-G400). Blood samples were collected in pre-test (fastening) and after 0.5, 1.0, 1.5, 2.0, 3.0 and 4.0 hours after the treatment ingested. Plasma obtained was used to measure glucose ([GLIC]), insulin ([INSU]) and total plasma protein ([TPP]) concentrations. Results were submitted to ANOVA with two factors (treatment and periods) and Tukey test, using SigmaPlot 13.0 software, with $P$ established in 5\%. It was observed differences between treatments and period in [GLIC] $(P<0.001)$, with significant interaction between treatment and phases $(P<0.044)$. Large [GLIC] was observed in T-GNa400 ( 113.8 mg/dL), followed by T-G400 ( 106,4 mg/dL) and T-GNa200 $(98,5 \mathrm{mg} / \mathrm{dL})(P<0.001)$. In addition, it was observed differences between treatments and in phases for [INSU] $(P<0.001)$. The interaction between treatment and period was observed for [INSU] $(P<0.037)$, and higher [INSU] was observed in T-GNa400 $(\sim 18.6 \mu \mathrm{IU} / \mathrm{mL})$, followed by T-G400 $(\sim 13.1 \mu \mathrm{IU} / \mathrm{mL})$ and T-GNa200 $(\sim 9.3 \mu \mathrm{IU} / \mathrm{mL})(P<0.001)$. It was not observed significante difference between treatments and phases in [TPP]. In conclusion, oral corn glucose test, with sodium, produced different curves for plasma glucose and insulin when compared with similar product without sodium in horses in maintenance, and after fastening, with large values detected in T-GNa400.

Keywords: Equine. Blood glucose. Hormone. Nutrition.

\section{Introdução}

Os equinos atletasparticipam devárias disciplinas equestres necessitando de um aporte regular de energia. Esses animais podem ser arraçoados com concentrados ricos em carboidratos não estruturais (CNE) presentes nos grãos de cereais, e/ou com gorduras, facilitando a reposição ou racionalizando as reservas de glicogênio hepático e muscular, que podem ser reduzidas durante os treinamentos e competições (Jansson et al., 2002; Frank et al., 2010). Devido as atuais práticas de arraçoamento, com o uso em larga escala de CNE como fontes de energia e oriundo dos grão de cereais processados (Jansson et al., 2002; Manso et al., 2015), os equinos passam a desenvolver com maior frequência problemas como obesidade, resistência à insulina, laminite, disfunções na microbiota intestinal e a síndrome metabólica equina (SMEq), principalmente com a maior longevidade nessa espécie (Malinowski et al., 2002; Lacombe, 2014).

Os equídeos são herbívoros típicos, e devido às características dos seus transportadores de glicose, poderão estar ou não adaptados ao uso em larga escala de CNE nos alimentos, comprometendo a regulação do metabolismo energético (Dyer et al., 2009; Waller et al., 2011). Esses transportadores são proteínas que facilitam o deslocamento das moléculas de glicose entre os tecidos e pertecem a dois grandes grupos: os dependentes do sódio 
(Na+-dependent glucose co-transporter ou SGLT) (Dyer et al., 2009; Daly et al., 2012) e os não dependentes do sódio (Na+-independent sugar transporter ou GLUT) (Manso Filho et al., 2007; Lacombe, 2014). Recentemente foi demonstrado que equinos suplementados com alimentos ricos em CNE apresentam elevação na expressão do SGLT1 e do GLUT-2 nos enterócitos quando comparados com animais que só recebem forragens (Daly et al., 2012). Esse processo favorece a elevação da glicose sanguínea no período pós-prandial nessa espécie, principalmente no cavalo atleta que é suplementado com alimentos ricos com CNE.

A síndrome metabólica dos equídeos (SMEq) acomete cavalos, pôneis, jumentos e muares, sendo associada ao tipo de manejo, com sobrealimentação e/ou a falta de exercícios, e principalmente pela suplementação excessiva com CNE (Malinowski et al., 2002; Frank, 2011; Morgan et al., 2015). 0 diagnóstico da SMEq é realizado através do uso de diferentes modelos de testes de glicose para formação da curva de glicose e insulina (Frank et al., 2010; Morgan et al., 2015; Ertelt et al., 2016). Esses testes ainda são indicados para o melhor entendimento dos efeitos dos diferentes alimentos sobre o metabolismo da glicose e sobre a microbiota intestinal (Manso et al., 2015).

Os testes de tolerância à glicose podem ser baseados no fornecimento oral de alimentos, ricos em carboidratos solúveis, com a glicose de milho ou similar, ou com testes de glicose intravenosos (Frank et al., 2010; Frank, 2011; Manso et al., 2015). Todavia, alguns investigadores demonstraram que o tipo de teste de glicose produz diferentes efeitos sobre o eixo entero-insular sobre a regulação da glicose, secreção da insulina e das increatinas (GLP-1 e GIP) nos equinos, (Graaf-Roelfsema, 2014; de Laat et al., 2015; Smith et al., 2016), reforçando a importância da caracterização dos testes orais com diferentes produtos para o diagnóstico e entendimento dos processos digestivos relacionados à SMEq. Para testar a hipótese de que a adição de sódio à glicose de milho produzirá curvas mais elevadas de glicose e insulina quando comparada com produto similar sem sódio, foram desenvolvidos experimentos com o objetivo de avaliar o teste oral de glicose com o uso da glicose de milho, com e sem sódio, sobre as concentrações plasmáticas de glicose, insulina e proteínas plasmáticas em equinos. Esperase que os resultados contribuam para o melhor entendimento desse processo e que favoreçam tanto a compreensão do processo digestivo dos carboidratos nos cavalos sadios, como ajudaem no diagnóstico da SMEq na espécie e por conseguinte, na adoção de melhores práticas de criação e manejo para os equídeos atletas.

\section{Material e métodos}

Todos os procedimentos utilizados no presente trabalho foram aprovados pela Comissão de Ética no Uso de Animais da Universidade Federal Rural de Pernambuco (CEUA-077/2016).

\section{Animais}

Foram utilizadas seis éguas da raça puro-sangue árabe, alojadas no Núcleo de Pesquisa Equina no Departamento de Zootecnia da Universidade Federal Rural de Pernambuco, Recife-PE. As éguas estavam em manutenção, clinicamente hígidas, idade média de 14+3 anos, escore corporal de 3,3 numa escala de 1 até 7 , e peso médio de $335 \mathrm{Kg}$. Esses animais eram mantidos em piquetes com pastagem variada (gramíneas e leguminosas) e suplementados com concentrado $(2,0 \mathrm{~kg} /$ dia/animal; proteína bruta $12 \%$, extrato etéreo 3,5\%, EB $2.6 \mathrm{Mcal} / \mathrm{Kg}$ ), água e sal mineralizado ad libitum.

\section{Tratamentos}

Os animais foram alocados com três tratamentos e seis éguas. Os tratamentos foram: 400 calorias de glicose de milho com sódio (glicose de milho Yoki ${ }^{\circledR}$ ) (T-GNa400), 200 calorias de glicose de milho com sódio (glicose de milho Yoki ${ }^{\circledR}$ ) (T-GNa200), e 400 calorias de glicose de milho sem sódio (glicose de milho Karo ${ }^{\circledR}$ ) (T-G400).

Amostras de sangue foram colhidas na veia jugular externa, com os animais em jejum alimentar de doze horas durante a noite, nas seguintes fases: pré-teste (jejum), e 0,5, 1,0, 1,5, 2,0, 3,0 e 4,0 horas após a ingestão do alimento. No dia da colheita de sangue, os animais receberam um dos tratamentos misturado em $25 \mathrm{~g}$ de farelo de trigo em comedouro individual para a ingestão voluntária do alimento em uma única vez. Eles não receberam alimentos 
durante toda a colheita de sangue, mas tiveram livre acesso a água.

Imediatamente após as colheitas de sangue, as amostras foram centrifugadas durante 5 minutos a 3.000rpm para a extração do plasma, que foi utilizado para a determinação das concentrações de glicose ([GLIC]) e das proteínas plasmáticas totais ([PPT]). Uma alíquota de plasma foi congelada $(-20 \mathrm{oC})$ para posterior análise da insulina. A concentração de glicose plasmática foi realizada através do glicosímetro portátil (Accu-Check ${ }^{\circledR}$, Roche do Brasil, São Paulo-SP, Brasil), e as proteínas plasmáticas totais pela refratometria manual. A concentração da insulina foi determinada pelo teste de ELISA (Human Insulin Elisa BioAssay Systems, CA, EUA), validado para equídeos (Barbosa et al., 2016).

Análise estatística: Os resultados foram submetidos ao ANOVA com dois fatores (três tratamentos e sete fases), e como teste post hoc foi utilizado o teste de Tukey, com o valor de $\mathrm{P}<0,05$, para todas as análises. 0 programa SigmaPlot 13.0 foi utilizado em todas as análises e os resultados estão expressos em média +/- erro padrão médio.

\section{Resultados}

Todos os animais ingeriram com mastigação uma das misturas de glicose de milho com farelo de trigo (50g) (tratamentos) em pouco menos de três minutos, e não apresentaram nenhum tipo de modificação clínica ou comportamental durante toda a fase de colheita de sangue. Os níveis de garantia da glicose de milho com e sem sódio estão descritos na Tabela 1.

Tabela 1 - Níveis de garantia da glicose de milho com e sem sódio

\begin{tabular}{lcc}
\hline Composição em 20g & Yoki $^{\circledast}$ & Karo \\
\hline Calorias & $65,0 \mathrm{cal}$ & $63,0 \mathrm{cal}$ \\
Açúcar total & $16,0 \mathrm{~g}$ & $16,0 \mathrm{~g}$ \\
Sódio & $16,0 \mathrm{mg}$ & $0,0 \mathrm{mg}$ \\
Gorduras totais & $0,0 \mathrm{~g}$ & $0,0 \mathrm{~g}$ \\
Fibra & $0,0 \mathrm{~g}$ & $0,0 \mathrm{~g}$ \\
\hline
\end{tabular}

Fonte: Yoki ${ }^{\circledR}$ : General Mills Brasil Alimentos Ltda, Paranavaí-PR; Karo ${ }^{\circledR}$ : Unilever Brasil Industrial Ltda, Garanhuns-PE.
Os resultados analisados pelo "2-way ANOVA" demonstram que a concentração de glicose difere tanto entre os tratamentos como entre as fases $(\mathrm{P}<0,001)$, e que há interação significativa entre os tratamentos e as fases( $\mathrm{P}=0,044)$ (Figura 1). A [GLIC] média foi diferente entre os tratamentos, sendo mais elevada no T-GNa400 (113,8 + 1,5 mg/ $\mathrm{dL})$, seguido pelo T-G400 $(106,4+1,5 \mathrm{mg} / \mathrm{dL})$ e mais baixa no T-GNa200 (98,5 + 1,5 mg/dL) $(\mathrm{P}<$ $0,001)$. As [GLIC] mais elevadas por tratamentos foram: $135,6+3,9 \mathrm{mg} / \mathrm{dL}$ em $+2 \mathrm{~h}$ no T-GNa400, $110,5+3,9 \mathrm{mg} / \mathrm{dL}$ em $+1 \mathrm{~h}$ no T-GNa200 e de 120,0 $+3,9 \mathrm{mg} / \mathrm{dL} \mathrm{em}+1,5 \mathrm{~h}$ no T-G400.
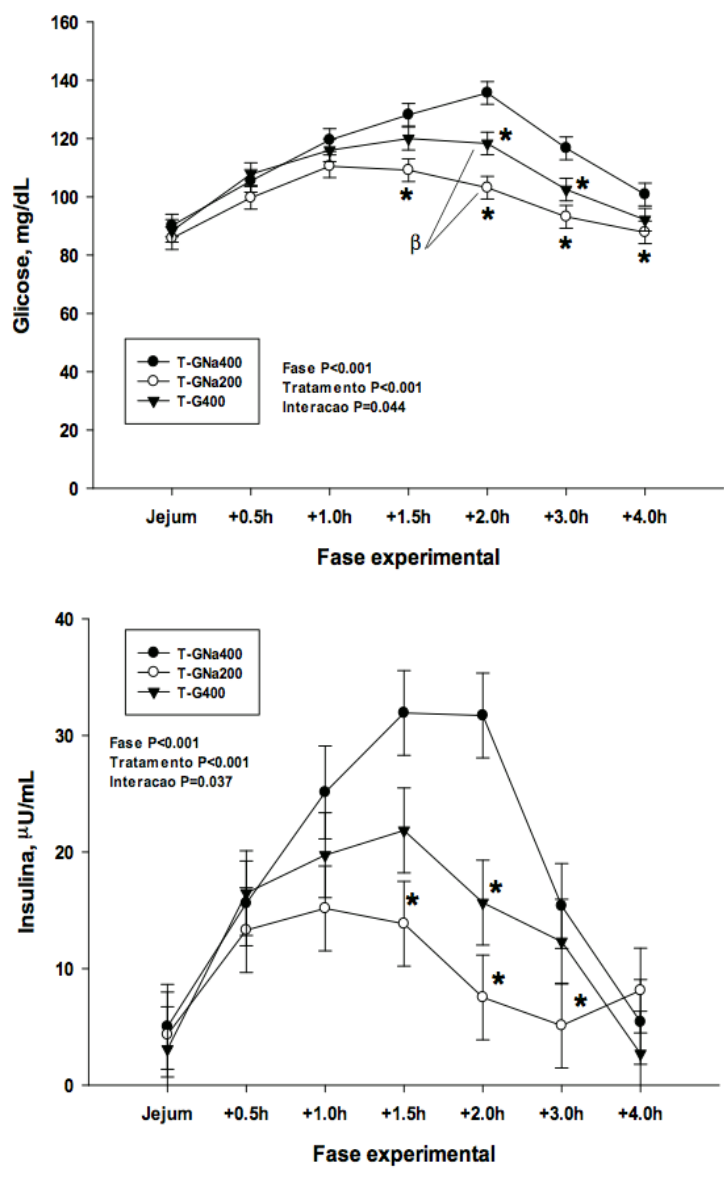

Figura 1 - Curvas das concentrações da glicose e da insulina nos animais suplementados com diferentes doses de glicose de milho com e sem sódio por via oral. Observações: *indica diferença do tratamento para o T-GNa400 $(P<0,05)$ no mesmo período experimental; $ß$ : indica que há diferença do T-G400 para o T-GNa200 $(P<0,05)$ no mesmo período experimental. 
As concentrações de insulina também demonstraram diferenças entre os tratamentos ( $\mathrm{P}$ $<0,001)$, entre as fases $(\mathrm{P}<0,001)$, e com interação significativa entre estes $(\mathrm{P}=0.037)$ (Figura 1$)$. A maior concentração de insulina foi observada no T-GNa400 $(18,59+1,39 \mu \mathrm{IU} / \mathrm{mL})$, seguido pelo T-G400 $(13,11+1,37 \mu \mathrm{IU} / \mathrm{mL})$ e pelo T-GNa200 $(9,63$ $+1,37 \mu \mathrm{IU} / \mathrm{mL})(\mathrm{P}<0,001)$. Na fase experimental, a maior concentração média foi com 1,5 hora $(22,54+$ $2,10 \mu \mathrm{IU} / \mathrm{mL}$ ) e a menor com os animais em jejum $(4,15+2,10 \mu \mathrm{IU} / \mathrm{mL})$. Quando avaliaram-se os tratamentos, as [INSU] mais elevadas foram: 31,71 $+3,64 \mu \mathrm{IU} / \mathrm{mL}$ em $+1,5 \mathrm{~h}$ no T-GNa400, de 15, 16 $+3,64 \mu \mathrm{IU} / \mathrm{mL}$ em $+1,0 \mathrm{~h}$ no T-GNa200 e de $21,85+$ $3,64 \mu \mathrm{IU} / \mathrm{mL}$ em $+1,5 \mathrm{~h}$ no T-G400.

Não ocorreram diferenças entre os tratamentos para a [PPT] média (T-GNa400: 68,55 + 0,08 g/L; T-G400: $67,79+0,08 \mathrm{~g} / \mathrm{L} ; \mathrm{T}-\mathrm{GNa} 200: 67,74$ $+0,08 \mathrm{~g} / \mathrm{L})(\mathrm{P}>0,05)$ e nem ao longo das fases experimentais ( $\mathrm{P}>0,05)$. A [PPT] foi de 69,67 + 0,11 $\mathrm{g} / \mathrm{L}$ quando os animais estavam em jejum, de 67,56 $+0,11 \mathrm{~g} / \mathrm{L}$ na fase $+2 \mathrm{~h}$, e de $66,50+0,11 \mathrm{~g} / \mathrm{L}$ ao final das 4 horas $(\mathrm{P}>0,05)$ (Figura 2).

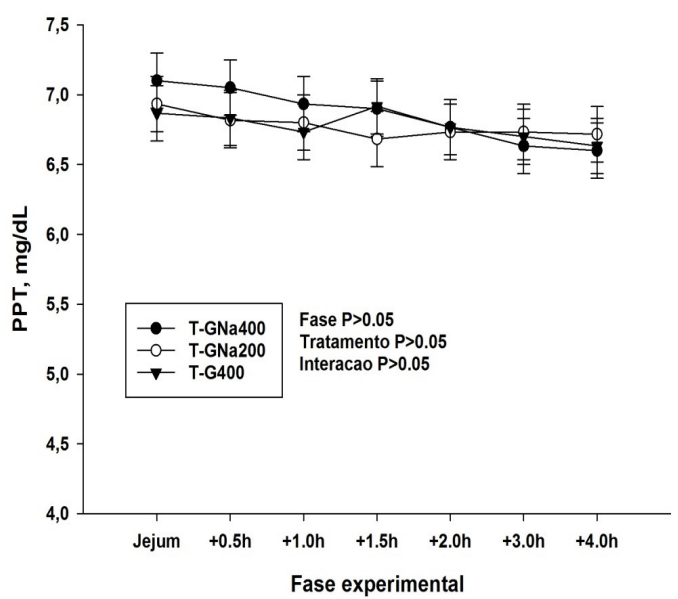

Figura 2 - Curva da proteína plasmática (PPT) total nos equinos suplementados com diferentes doses com glicose de milho com e sem sódio via oral.

\section{Discussão}

Os resultados demonstraram que não é possível obter padrões semelhantes entre as curvas de glicose e de insulina utilizando-se glicose de milho com e sem sódio, que estão comercialmente disponíveis no Brasil, já que a presença desse mineral favorece a absorção da glicose pelo SGLT-1, presente nos enterócitos. Os desafios propostos no atual experimento demonstraram curvas diferentes entre os produtos, tanto para as concentrações de glicose como para as de insulina, mesmo quando os animais receberam a mesma quantidade de calorias. Esse fato é importante para os pesquisadores e técnicos no Brasil quando utilizarem o teste oral de glicose com ingestão voluntária como meio de diagnóstico da SMEq, seguindo as indicações da literatura internacional (Frank, 2010; Frank et al., 2011). Ressalta-se que os animais estavam sob o mesmo manejo nutricional desde longo tempo, e por isso apresentavam adaptações semelhantes no aparelho digestivo.

No atual experimento foram utilizados dois tipos de glicose de milho, um com sódio e outro sem, e acredita-se que os resultados encontrados refletem a maior eficiência da absorção da glicose presente no T-GNa400, que contem sódio, sobre a do T-G400. Esse fato está relacionado com a maior captação da glicose, juntamente com o sódio, através do SGLT-1 pelos enterócitos, seguido do transporte da glicose pelo GLUT-2 para a corrente sanguínea do cavalo. Esse fato ainda é corroborado quando se compara o T-G400 e o T-GNa200, este fornecendo a metade das colorias sob a forma de glicose do que o anterior. Ambos apresentaram resultados semelhantes tanto para a glicose como para a insulina entre si, só apresentando diferenças significativas na fase + 2horas para a glicose (T-G400: 118 mg/dL; T-GNa200: 109 mg/dL), o que não era esperado devido a diferença na quantidade de calorias entre os dois tratamentos.

Nos equinos a expressão do cotransportador SGLT-1 tem sido estudada para melhorar a compreensão da absorção da glicose nos enterócitos nesta espécie. O SGLT-1 está relacionado com a captação de glicose nas vilosidades dos enterócitos e a sua maior ou menor expressão está relacionada com o tipo de alimentação dos animais (Dyer et al., 2009). A expressão do SGLT-1 e do GLUT-2 nos enterócitos está reduzida nos cavalos alimentados exclusivamente com forragens quando comparados com os que recebem concentrados ricos em 
carboidratos não estruturais (CNE) (Dyer et al., 2009). Esses pesquisadores ainda indicam que o aumento da expressão do SGLT-1 e do GLUT-2, após um período de adaptação, favorece a captação da glicose pelos enterócitos, reduzindo a passagem de CNE ou glicose para a fermentação no ceco e colo. Por isso, quando animais são submetidos aos testes de tolerância à glicose, deve-se esperar resultados compatíveis com o tipo de alimentação que eles estão recebendo antes do teste.

Ainda, analisando a importância dos transportadores de glicose ligados ao sódio sobre o processo digestivo, foi demonstrado que o SGLT1, o SGLT-2 e o SGLT-3 servem como sensores da presença de glicose e de algumas gorduras ao longo do aparelho digestivo, sendo importantes para o signaling da absorção da glicose e de lipídeos nas fases pré-prandial (Diez-Sampedro et al., 2003; Daly et al., 2012). Nesse aspecto, o eixo enteroinsular nos equinos, que está relacionado com esses cotransportadores, faz com que nos testes orais de glicose ocorram em formação de curvas de glicose, insulina, GLP-1 e GIP diferentes quando comparados com os testes intravenosos de glicose (Dühlmeier et al., 2001; Daly et al., 2012; de Laat et al., 2015).

Finalmente, deve-se ressaltar a importância dos testes orais de glicose para o entendimento da digestão da glicose nos equinos e em outros processos. Os resultados encontrados na atual avaliação assemelham-se com outros descritos na literatura, com grande elevação nas concentrações de glicose e insulina cerca de 2 a 3 horas após o teste oral com glicose (Ralston, 2002; Frank, 2010); todavia, deve-se recordar que a variação na curva, além de depender da quantidade de calorias ingeridas, depende da quantidade de CNE presente no alimento. Sendo assim, o teste oral com ingestão voluntária representa mais claramente os processos fisiológicos da digestão, como por exemplo as ações das incretinas GLP-1 e GIP após o fornecimento de alimentos para os equídeos, e pode ser facilmente replicado pelos pesquisadores e técnicos.

\section{Conclusão}

Analisando-se os resultados encontrados, conclui-se que a glicose de milho, com sódio, durante o teste oral com ingestão, produz curvas sanguíneas de glicose e insulina diferentes da glicose de milho sem sódio, quando os equinos estão em jejum e recebem a mesma quantidade de calorias, sendo mais elevadas no T-GNa400. Esses resultados eram esperados pois o SGLT-1, presente no intestino delgado torna mais eficiente a absorção da glicose, por conseguinte, ocasiona maiores modificações nas curvas de glicose e insulina plasmáticas nos animais.

\section{Referências}

Barbosa BL, Silva GB, Silva CJFL, Ferreira LMC, Manso HECCC, Manso Filho HC. Blod metabolic and endocrine biomarkers in donkeys (Equus africanus asinus) supplemented with different energy sources. Acta Veterinaria Brasilica, 2016;10(2):135-43.

Daly K, Al-Rammahi M, Arora DK, Moran AW, Proudman CJ, Ninomiya $Y$, et al. Expression of sweet receptor components in equine small intestine: relevance to intestinal glucose transport. Am J Physiol Regul Integr Comp Physiol. 2012;303(2):R199-208.

Dühlmeier R, Deegen E, Fuhrmann H, Widdel A, Sallmann H-P. Glucose-dependent insulinotropic polypeptide (GIP) and the enteroinsular axis in equines (Equuscaballus). Comp Biochem Physiol Part A MolIntegr Physiol. 2001;129(2):563-75.

Dyer J, Al-Rammahi M, Waterfall L, Salmon KSH, Geor RJ, Bouré $\mathrm{L}$, et al. Adaptive response of equine intestinal $\mathrm{Na}+$ /glucose co-transporter (SGLT1) to an increase in dietary soluble carbohydrate. Pflugers Arch. 2009;458(2):419-30.

Ertelt A, Barton A-K, Schmitz RR, Gehlen H. Metabolic syndrome: is equine disease comparable to what we know in humans? Endocr Connect. 2016;3(3):R81-93.

Frank N, Geor RJ, Bailey SR, Durham AE, Johnson PJ; American College of Veterinary Internal Medicine. Equine metabolic syndrome. J Vet Intern Med. 2010;24(3):467-75.

Frank N. Equine metabolic syndrome. Vet Clin North Am Equine Pract. 2011;27(1):73-92. 
Graaf-Roelfsema E. Glucose homeostasis and the enteroinsular axis in the horse: a possible role in equine metabolic syndrome. Vet J. 2014;199(1):11-8.

Jansson A, Nyman S, Lindholm A, Lindberg JE. Effects on exercise metabolism of varying dietary starch and sugar proportions. Equine Vet J Suppl. 2002;(34):17-21.

Laat MA, McGree JM, Sillence MN. Equine hyperinsulinemia: investigation of the enteroinsular axis during insulin dysregulation. Am J Physiol Endocrinol Metab. 2016;310(1):E61-72.

Lacombe VA. Expression and regulation of facilitative glucose transporters in equine insulin-sensitive tissue: from physiology to pathology. ISRN Vet Sci. 2014;2014:409547.

Malinowski K, Betros CL, Flora L, Kearns CF, McKeever $\mathrm{KH}$. Effect of training on age-related changes in plasma insulin and glucose. Equine Vet J Suppl. 2002;(34):147-53.

Manso HECCC, Silva AEM, Santiago TA, Almeida TLAC, Duarte SR, Manso Filho HC. Biomarcadores sanguíneos de cavalos consumindo milho processado de diferentes formas ou farelo de algaroba. Rev Acad Cienc Anim. 2015;13:69-77.

Manso Filho HC, McKeever KH, Gordon ME, Costa HEC, Watford M. Novel findings regarding Glut-4 expression in adipose tissue and muscle in horses - A preliminary report. Vet J. 2007;174(3):565-9.

Morgan RA, Keen JA, McGowan CM. Treatment of equine metabolic syndrome: A clinical case series. Equine Vet J. 2016;48(4):422-6.

Ralston SL. Insulin and glucose regulation. Vet Clin North Am Equine Pract. 2002;18(2):295-304.

Smith S, Harris PA, Menzies-Gow NJ. Comparison of the in-feed glucose test and the oral sugar test. Equine Vet J. 2016;48(2):224-7.

Waller AP, Burns TA, Mudge MC, Belknap JK, Lacombe

Recebido em: $14 / 12 / 2016$

Received in: 12/14/2016

Glucose Transporters but not their Total Protein Expression in Equine Skeletal Muscle. J Vet Intern Med.

Aprovado em: 12/05/2017

2011;25(2):315-21.

Approved in: 05/12/2017 